\title{
Effect of Constant Heat Flux on Forced Convective Micropolar Fluid Flow over a Surface of Another Quiescent Fluid
}

\author{
Nurazleen Abdul Majid ${ }^{1}$, Nurul Farahain Mohammad ${ }^{1, *}$, Abdul Rahman Mohd Kasim $^{2}$, \\ Mohd Rijal Ilias ${ }^{3}$, Sharidan Shafie ${ }^{4}$ \\ ${ }^{1}$ Department of Computational and Theoretical Sciences, Kulliyyah of Science, International Islamic University Malaysia, \\ 25200 Pahang, Malaysia. \\ ${ }^{2}$ Applied \& Industrial Mathematics Research Group, Faculty of Industrial Sciences \& Technology, Universiti Malaysia Pahang, \\ 26300 Gambang, Kuantan, Pahang, Malaysia. \\ ${ }^{3}$ Department of Mathematical Sciences, Faculty of Computer and Mathematical Sciences, UiTM Shah Alam, \\ 40450 Shah Alam, Selangor Malaysia \\ ${ }^{4}$ Department of Mathematical Sciences, Faculty of Science, Universiti Teknologi Malaysia, 81310 Skudai, Johor, Malaysia.
}

Copyright (C) 2019 by authors, all rights reserved. Authors agree that this article remains permanently open access under the terms of the Creative Commons Attribution License 4.0 International License

\begin{abstract}
Due to the many applications of micropolar fluid such as blood, paint, body fluid, polymers, colloidal fl uid and suspension fluid, it has become a prominent subject among the researchers. However, the characteristics of micropolar fluid flow over a surface of another quiescent fluid with heavier density of micropolar fluid under the effect of constant heat flux is still unknown. Therefore, the objective of the present work is to investigate numerically the forced convection of micropolar fluid fl ow over a su rface of an other qu iescent flu id using constant heat flux boundary c ondition. In this study, the similarity transformation is used to reduce the boundary layer governing equations for mass, momentum, angular momentum and energy from partial differential equations to a system of nonlinear ordinary differential equations. This problem is solved numerically using shooting technique with Runge-Kutta-Gill method and implemented in Jupyter Notebook using Python 3 language. The behaviour of micropolar fluid in terms of velocity, skin friction, microrotation and temperature are analyzed and discussed. It is found that, the temperature is higher in constant wall temperature (CWT) compared to constant heat flux (CHF) at stretching or shrinking parameter $\lambda=0.5$ and various micropolar parameter $K$. Furthermore, as Prandtl num-ber increases, the temperature is decreasing in both CHF and CWT.
\end{abstract}

Keywords Micropolar Fluid, Forced Convection, Constant Heat Flux, Quiescent Fluid

\section{Introduction}

Eringen [1] was the first who propose the theory of micropolar fluid to extend the widely known equation of viscous fluid which is Navier-Stokes equation. Micropolar fluid can be defined as a non-Newtonian fluid that consist of microstructure which undergo translational motion and rotational motion. In addition, this theory also capable to describe some fluid phenomena that contain suspended fluid additives such as animal blood, body fluid, colloidal fluid, magnetic fluids, cloud with dust, muddy fluids and polymer that cannot be interpreted by Navier-Stokes equation. A comprehensive information about micropolar fluid can be found in papers by Ariman et. al [2] - [3], Willson [4] and books written by Lukaszewicz [5] and Eremeyev et. al [6]. Due to the extensive applications, micropolar fluid has been studied on different geometry and system by various researchers such as Rees and Bassom [7], Guram and Smith [8], Peddieson [9], Takhar and Soundalgekar [10], Ishak et. al [11] and Das \& Guha [12].

The problem of heat transfer in boundary layer flow is one of the growing research field as it has numerous applications such as transpiration cooling, drag reduction, bearing and radial diffusers, thermal recovery of oil, the design of thrust bearings and radial diffusers, material drying, laser pulse heating and more [13]. Eringen extended the problem of micropolar fluid with addition of thermal effect [14] . Gorla et al. [15] studied the heat transfer in micropolar boundary layer flow over a flat plate for both constant wall temperature and constant heat flux. Ishak et al. [16] discussed the effect of constant surface heat flux on boundary layer flow of a micropolar fluid on a continuous flat plate moving in a parallel stream. They concluded 
micropolar fluid exhibit drag reduction compared to Newtonian fluid and delayed the boundary layer separation.

However, there are some systems that exist with two fluids involved. Thus, several attempts have been made in the case of another quiescent fluid such as Wang [17] who investigated the stagnation flow on the surface of a quiescent fluid. Next, Liu [18] analyzed the nonorthogonal stagnation flow on the surface of a quiescent fluid. Reza et al. [19] carried out the numerical study of stagnation point flow and heat transfer for viscoelastic fluid impinging on a quiescent fluid. The magnetic effect on the electrically conducting fluid in the surface of another quiescent fluid was attempted by Reza \& Gupta [20]. Rohini et al. [21] examined stagnation-point flow of a fluid on a shrinking surface of another quiescent fluid. More recently, Isa and Mohammad [22] performed numerical analysis of boundary layer flow on a stretching sheet of another quiescent fluid. Although some research were carried out on another quiescent fluid, there is no study involve micropolar fluid in context of convective flow within our knowledge. Therefore, the aim of this paper is to investigate the effect of constant heat flux (CHF) and constant wall temperature (CWT) on forced convective micropolar fluid flow over a surface of another quiescent fluid.

\section{Mathematical Model}

Consider an incompressible micropolar fluid of density $\rho_{1}$, dynamic viscosity $\mu_{1}$, vortex viscosity $\kappa_{1}$, spin-gradient viscosity $\gamma_{1}$ and microinertia density $j_{1}$ impinging orthogonally on a surface of another quiescent, heavier incompressible micropolar fluid of density $\rho_{2}$, dynamic viscosity $\mu_{2}$, vortex viscosity $\kappa_{2}$, spin-gradient viscosity $\gamma_{2}$ and microinertia density $j_{2}$. $T_{1, w}, T_{2, w}$ and $T_{\infty}$ are temperature of surface for upper fluid, lower fluid and free stream temperature respectively. A sketch of the physical problem is shown in Figure 1. Let $\left(x, y_{1}\right)$ denote the Cartesian coordinates for the upper fluid with $x=0$ as the symmetry plane, and $x$-axis is taken along the interface between the two fluids. It is assumed that the surface is stretched or shrinked with velocity $u_{w}(x)=c x$, where $c$ is a constant that indicates $c>0$ for a stretching sheet and $c<0$ for a shrinking sheet, respectively. The coordinate system for the lower fluid is $\left(x, y_{2}\right)$ as shown in Figure 1. Note that the $z$-axis is normal to the $\left(x, y_{1}\right)$ plane.

Under the boundary layer approximations, the governing equations of continuity, momentum, angular momentum and energy are,

$$
\begin{gathered}
\frac{\partial u_{i}}{\partial x}+\frac{\partial v_{i}}{\partial y_{i}}=0 \\
u_{i} \frac{\partial u_{i}}{\partial x}+v_{i} \frac{\partial u_{i}}{\partial y_{i}}=U_{i} \frac{d U_{i}}{d x}+\frac{\mu_{i}+\kappa_{i}}{\rho_{i}} \frac{\partial^{2} u_{i}}{\partial y_{i}^{2}}+\frac{\kappa_{i}}{\rho_{i}} \frac{\partial N_{i}}{\partial y_{i}} \\
\rho_{i} j_{i}\left(u_{i} \frac{\partial N_{i}}{\partial x}+v_{i} \frac{\partial N_{i}}{\partial y_{i}}\right)=\gamma_{i} \frac{\partial^{2} N_{i}}{\partial y_{i}^{2}}-\kappa_{i}\left(2 N_{i}+\frac{\partial u_{i}}{\partial y_{i}}\right)
\end{gathered}
$$$$
u_{i} \frac{\partial T_{i}}{\partial x}+v_{i} \frac{\partial T_{i}}{\partial y_{i}}=\frac{k_{0}}{\rho_{i} c_{p}} \frac{\partial^{2} T_{i}}{\partial y_{i}^{2}},
$$

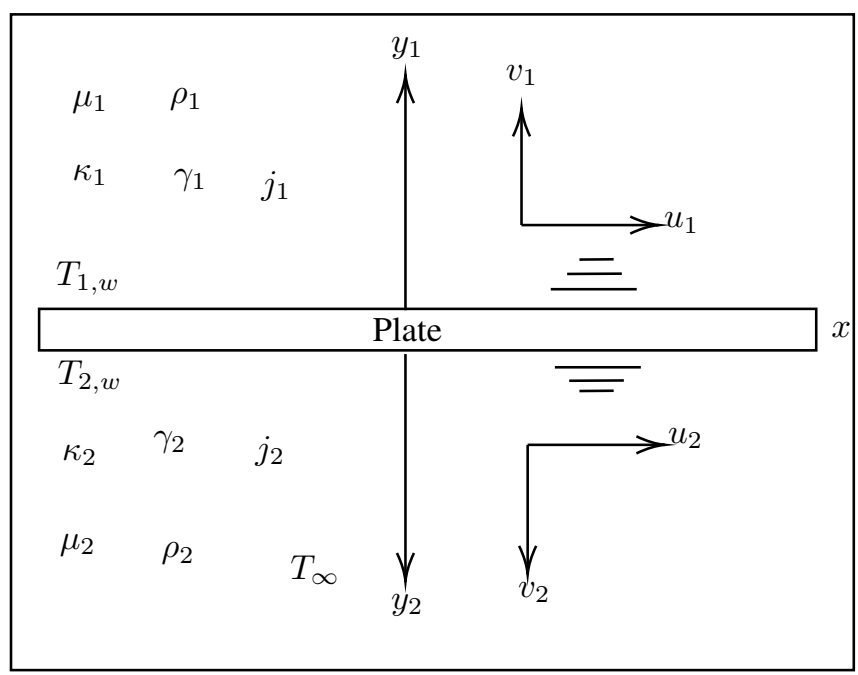

Figure 1. Physical coordinate.

$$
\begin{gathered}
u_{i}(x)=c x, v_{i}=0, N_{i}=-n \frac{\partial u_{i}}{\partial y_{i}} \text { at } y_{i}=0, \\
u_{1}(x) \rightarrow a x, u_{2}(x) \rightarrow 0, N_{i} \rightarrow 0 \text { as } y_{i} \rightarrow \infty,
\end{gathered}
$$

for temperature, we have

$$
\frac{\partial T_{i}}{\partial y_{i}}=\frac{-q_{w}}{k_{0}}(\mathrm{CHF}) \text { at } y_{i}=0, T_{i} \rightarrow T_{\infty},
$$

or

$$
T_{i}=T_{i, w}(\mathrm{CWT}) \text { at } y_{i}=0, T_{i} \rightarrow T_{\infty},
$$

where $u_{i}$ and $v_{i}$ are the velocity along $x$ and $y_{i}$ axes, $U_{i}$ is free stream velocity, $T_{i}$ is the temperature and $N_{i}$ is the angular velocity. $i=1$ represents upper fluid while $i=2$ represents lower fluid. Moreover, $q_{w}$ is constant heat flux, $k_{0}$ is thermal conductivity, $c_{p}$ is specific heat capacity and $n$ is a constant such that $0 \leq n \leq 1$. From Jena and Mathur [23], the strong concentration case $(n=0)$ represents the concentrated particle flows in which the microelements close to the wall surface are unable to rotate. According to Ahmadi [24], the weak concentration case $(n=1 / 2)$ indicates the vanishing of the anti-symmetrical part of the stress tensor. The case $n=1$, as suggested by Peddieson [9], is used for the modeling of turbulent boundary layer flows. In this paper, we consider the cases of $n=0$ (strong concentration) only and it is worth to mention case $K=0$ represent the classical Navier-Stokes equations for a viscous and incompresible fluid. Further, we assume that spin-gradient viscosity $\gamma_{i}$ is defined as $[25,26,27]$

$$
\gamma_{i}=\left(u_{i}+\kappa_{i} / 2\right) j_{i}=\mu_{i}(1+K / 2) j_{i}
$$

Following Attia [13] for the upper fluid, the similarity variables are

$$
\begin{aligned}
& u_{1}=a x f^{\prime}(\eta), v_{1}=-\sqrt{a \nu_{1}} f(\eta), N_{1}=a x \sqrt{a / \nu_{1}} g(\eta) \\
& \eta=y_{1} / \sqrt{\nu_{1} / a}
\end{aligned}
$$


for temperature distribution, we have

$$
\theta_{1}=\frac{k\left(T_{1}-T_{\infty}\right)}{q_{w}}\left(\sqrt{\frac{a}{\nu_{1}}}\right)(\mathrm{CHF}[13])
$$

or

$$
\theta_{1}=\frac{T_{1}-T_{\infty}}{T_{1, w}-T_{\infty}}(\mathrm{CWT}[16]),
$$

and for lower fluid

$$
\begin{aligned}
& u_{2}=a x F^{\prime}(\xi), v_{2}=-\sqrt{a \nu_{2}} F(\xi), N_{2}=a x \sqrt{a / \nu_{2}} G(\xi) \\
& \xi=y_{2} / \sqrt{\nu_{2} / a} .
\end{aligned}
$$

for temperature distribution,

$$
\theta_{2}=\frac{k\left(T_{2}-T_{\infty}\right)}{q_{w}}\left(\sqrt{\frac{a}{\nu_{2}}}\right)
$$

or

$$
\theta_{2}=\frac{T_{2}-T_{\infty}}{T_{2, w}-T_{\infty}}
$$

where prime denotes differentiation with respect to $\eta$ and $\xi$ respectively. Clearly with $u_{1}$ and $v_{1}$ given in (10), the equation of continuity (1) is satisfied. Similarly, for the lower fluid, with $u_{2}$ and $v_{2}$ given as in (13), it is readily seen that the continuity equation (1) is identically satisfied. Using (10) - (15), equations (2) - (8) are transformed into ordinary differential equations for the upper fluid flow

$$
\begin{gathered}
\left(1+K_{1}\right) f^{\prime \prime \prime}+f f^{\prime \prime}-f^{\prime 2}+K_{1} g^{\prime}+1=0, \\
\left(1+K_{1} / 2\right) g^{\prime \prime}+f g^{\prime}-f^{\prime} g-K_{1}\left(2 g^{\prime}+f^{\prime \prime}\right)=0, \\
\theta_{1}^{\prime \prime}+\operatorname{Pr} f \theta_{1}^{\prime}=0,
\end{gathered}
$$

with the boundary conditions

$$
\begin{aligned}
& f(0)=0, f^{\prime}(0)=\lambda, g(0)=-n f^{\prime \prime}(0), \\
& f^{\prime}(\eta) \rightarrow 1, \quad g(\eta) \rightarrow 0, \quad \theta_{1}(\eta) \rightarrow 0 \text { as } \eta \rightarrow \infty .
\end{aligned}
$$

On the other hand, for the lower fluid, we obtain

$$
\begin{gathered}
\left(1+K_{2}\right) F^{\prime \prime \prime}+F F^{\prime \prime}-F^{2}+K_{2} G^{\prime}=0, \\
\left(1+K_{2} / 2\right) G^{\prime \prime}+F G^{\prime}-F^{\prime} G-K_{2}\left(2 G^{\prime}+F^{\prime \prime}\right)=0, \\
\theta_{2}^{\prime \prime}+\operatorname{Pr} f \theta_{2}^{\prime}=0,
\end{gathered}
$$

with the boundary conditions

$$
\begin{aligned}
& F(0)=0, F^{\prime}(0)=\lambda, G(0)=-n F^{\prime \prime}(0), \\
& F^{\prime}(\xi) \rightarrow 0, \quad G(\xi) \rightarrow 0, \quad \theta_{2}(\xi) \rightarrow 0 \text { as } \xi \rightarrow \infty .
\end{aligned}
$$

where Pr is Prandlt number, $\lambda$ is stretching or shrinking parameter, $K_{1}$ and $K_{2}$ are micropolar parameters. These quantities can be written as

$$
\operatorname{Pr}=\frac{c_{p} \mu_{i}}{\kappa_{i}}, \lambda=\frac{c}{a}, K_{i}=\frac{\kappa_{i}}{\mu_{i}}, i=1,2 .
$$

In the case of constant heat flux, the initial conditions for temperature are

$$
\theta_{1}^{\prime}(0)=-1, \theta_{2}^{\prime}(0)=-1(\mathrm{CHF})
$$

whilst, in the case of constant wall temperature, the initial conditions for temperature are

$$
\theta_{1}(0)=1, \theta_{2}(0)=1(\mathrm{CWT}) .
$$

In addition, the local Nusselt number $N u_{x}$ can be denoted in non-dimensional form as

$$
N u_{x}=\frac{x q_{w}}{k_{0}\left(T_{w}-T_{\infty}\right)},
$$

and $q_{w}$ can be defined as

$$
q_{w}=-\left.k_{0}\left(\frac{\partial T_{i}}{\partial y_{i}}\right)\right|_{y_{i}=0}
$$

Next using (11) - (12), (27) and (28), for upper fluid we attained

$$
\begin{aligned}
& \frac{N u_{x}}{R e_{x}^{1 / 2}}=\frac{1}{\theta_{1}(0)}(\mathrm{CHF}), \\
& \frac{N u_{x}}{R e_{x}^{1 / 2}}=-\theta_{1}^{\prime}(0)(\mathrm{CWT}) .
\end{aligned}
$$

Furthermore, using equations (14), (15), (27) and (28), for lower fluid we obtained

$$
\begin{aligned}
& \frac{N u_{x}}{R e_{x}^{1 / 2}}=\frac{1}{\theta_{2}(0)}(\mathrm{CHF}) \\
& \frac{N u_{x}}{R e_{x}^{1 / 2}}=-\theta_{2}^{\prime}(0)(\mathrm{CWT}) .
\end{aligned}
$$

\section{Results and Discussion}

In this study, the system of non-linear ordinary differential equations with boundary conditions (16) - (23) and (25) - (26) were solved using shooting technique with Runge-Kutta-Gill method. The numerical procedure was implemented in Jupyter Notebook using Python 3 language. The results presented are in term of velocity, skin friction, microrotation and temperature of the micropolar fluids in strong concentration $(n=0)$ where the micropolar parameter for both upper and lower fluids are the same $\left(K=K_{1}=K_{2}\right)$. The solutions obtained involve initial guess of $f^{\prime}(0), g^{\prime}(0)$ and $\theta(0)$ for CHF case and $f^{\prime}(0)$, $g^{\prime}(0)$ and $\theta^{\prime}(0)$ for CWT case. Then the Newton-Raphson method is used to find the correct initial conditions that satisfy the boundary conditions. The comparison of the solutions obtained in current study with existing work for wall temperature $\theta(0)$ and heat transfer coefficient $\theta^{\prime}(0)$ are depicted in table 1 and table 2 respectively. In these tables, the temperature at surface and the heat transfer at surface from current study are found in a good agreement with Lok et al. [28] and Salleh et al. [29]. Practically, small values of $\operatorname{Pr}(<1)$ resemble liquid metal that have low viscosity but high thermal conductivity, meanwhile large values of $\operatorname{Pr}(>1)$ equivalent to high viscosity. In addition, Prandtl number $\operatorname{Pr}=0.71,1,7$ represent air, electrolyte solution and water, respectively [29].

The graphical results for velocity, temperature and microrotation profiles for upper and lower fluids are illustrated in figures 2 - 7 for various micropolar parameter $K$ with $\operatorname{Pr}=7$ and 
Table 1. Comparison of temperature at surface $\theta(0)$ with various $\operatorname{Pr}$ when $K=0$ for CHF.

\begin{tabular}{|l|l|ll|}
\hline \multirow{2}{*}{$\operatorname{Pr}$} & \multicolumn{3}{|c|}{$\theta(0)(\mathrm{CHF})$} \\
\cline { 2 - 4 } & Lok et al. [28] & Salleh et al. [29] & Present \\
\hline 0.01 & 13.1627 & 13.1209 & 13.1209 \\
0.02 & 9.4844 & 9.4841 & 9.4841 \\
0.04 & 6.8785 & 6.8785 & 6.8785 \\
0.06 & 5.7209 & 5.7209 & 5.7209 \\
0.08 & 5.0290 & 5.0290 & 5.0290 \\
0.1 & 4.5557 & 4.5557 & 4.5557 \\
0.2 & 3.3743 & 3.3742 & 3.3743 \\
0.4 & 2.5267 & 2.5267 & 2.5268 \\
0.6 & 2.1444 & 2.1444 & 2.1444 \\
0.7 & 2.0166 & 2.0166 & 2.0167 \\
0.8 & 1.9130 & 1.9130 & 1.9130 \\
1 & 1.7529 & 1.7529 & 1.7530 \\
5 & 0.9583 & 0.9583 & 0.9584 \\
7 & 0.8485 & 0.8485 & 0.8486 \\
10 & 0.7468 & 0.7468 & 0.7469 \\
\hline
\end{tabular}

stretching parameter $\lambda=0.5$. Based on figure 2 , the velocity of upper fluid is decreasing and the boundary layer thickness is increasing as micropolar parameter $K$ increase. From figure 3 , the velocity of lower fluid is increasing as micropolar parameter $K$ increases. Furthermore, from table 3, we observe that increasing micropolar parameter $K$ lead to decrease in skin friction resulting less velocity for upper fluid. However, from the same table, skin friction is increasing with the increament of micropolar parameter $K$ for lower fluid. We also notice that the values of skin friction is similar for both $\mathrm{CWT}$ and $\mathrm{CHF}$ due to decouple governing equations. Refering to figure 4 , the microrotation of upper fluid is decreasing with the addition of micropolar parameter $K$. It is also has a profile that achieving the maximum value nearby the wall which later declining to zero as boundary layer increases. In figure 5, the microrotation of lower fluid is increasing as micropolar parameter $K$ increases. Meanwhile figure 6 shows the temperature of upper fluid is decreasing when micropolar parameter $K$ increases. Also, the temperature of upper fluid is higher under the influence of CWT boundary condition compared to CHF. On the other hand, the temperature of lower fluid is increasing with the increament of micropolar parameter $K$ as displayed in figure 7. It seem that upper fluid and lower fluid display opposite characteristic.

Figures 8 - 13 show the velocity profiles, temperature profiles and microrotation respectively for various Pr with $K=1$, $n=0$ and $\lambda=0.5$ for both upper and lower fluid. From figures 8 - 11, we can see that changes in Pr does not influence the movement of velocity and microrotation for upper fluid and lower fluid due to the decouple governing equations in (16) (17) and (18) as mentioned before. In figure 12, we found that the temperature of upper fluid is higher in CWT compared to $\mathrm{CHF}$ but as Pr increases the values of temperature decrease. Increasing Pr means increasing the viscosity of the fluid which
Table 2. Comparison of heat transfer at surface $-\theta^{\prime}(0)$ with various $\operatorname{Pr}$ when $K=0$ for CWT.

\begin{tabular}{|l|l|l|l|}
\hline \multirow{2}{*}{$\operatorname{Pr}$} & \multicolumn{3}{|c|}{$-\theta^{\prime}(0)(\mathrm{CWT})$} \\
\cline { 2 - 4 } & Eckert [30] & Salleh et al. [29] & Present \\
\hline 0.01 & - & 0.0761 & 0.0762 \\
0.02 & - & 0.1061 & 0.1054 \\
0.04 & - & 0.1454 & 0.1454 \\
0.06 & - & 0.1748 & 0.1748 \\
0.08 & - & 0.1988 & 0.1988 \\
0.1 & - & 0.2195 & 0.2195 \\
0.2 & - & 0.2964 & 0.2964 \\
0.4 & - & 0.3958 & 0.3958 \\
0.6 & - & 0.4663 & 0.4663 \\
0.7 & 0.496 & 0.4959 & 0.4959 \\
0.8 & 0.523 & 0.5228 & 0.5227 \\
1 & 0.570 & 0.5705 & 0.5705 \\
5 & 1.043 & 1.0436 & 1.0434 \\
7 & - & 1.1786 & 1.1784 \\
10 & 1.344 & 1.3391 & 1.3388 \\
\hline
\end{tabular}

Table 3. Skin friction $f^{\prime \prime}(0) \& F^{\prime \prime}(0)$ for various value of $K$ when $\operatorname{Pr}=7$ for CWT and CHF cases.

\begin{tabular}{|l|lllll|}
\hline \multirow{2}{*}{$\mathrm{K}$} & \multicolumn{4}{|l|}{ Upper fluid $f^{\prime \prime}(0)$} & \multicolumn{2}{l|}{ Lower fluid $F^{\prime \prime}(0)$} \\
\cline { 2 - 5 } & CWT & CHF & CWT & CHF \\
\hline 0 & 0.7133 & 0.7133 & -0.3536 & -0.3536 \\
\hline 1 & 0.4905 & 0.4905 & -0.2386 & -0.2386 \\
\hline 2 & 0.3877 & 0.3877 & -0.1872 & -0.1872 \\
\hline
\end{tabular}

lead to less heat and the fluid is highly conductive when Pr number is small. It is seen that thermal boundary layer thickness is greater for CHF compared to CWT and has decreasing trend as Pr number increase. The thermal diffusivity decreases as Pr number increase consequently lead to the decreasing of energy transfer capability that abbreviates the thermal boundary layer thickness. Figure 13 also potrays similar trend. It is seen that for CHF, lower fluid has greater temperature at surface and thermal boundary layer thickness.



Figure 2. Velocity $f^{\prime}(\eta)$ of upper fluid for various $K$ when $\operatorname{Pr}=7$ and $\lambda=0.5$ under the effect of $\mathrm{CHF}$ 




Figure 3. Velocity $F^{\prime}(\xi)$ of lower fluid for various $K$ when $\operatorname{Pr}=7$ and $\lambda=0.5$ under the effect of CHF.

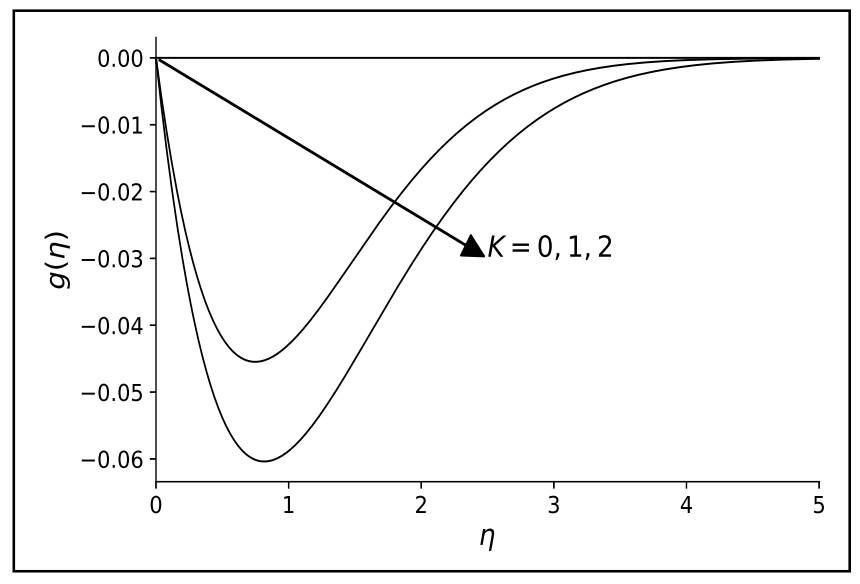

Figure 4. Microrotation profiles $g(\eta)$ of upper fluid for various $K$ when $\operatorname{Pr}=$ 7 and $\lambda=0.5$ under the effect of CHF.

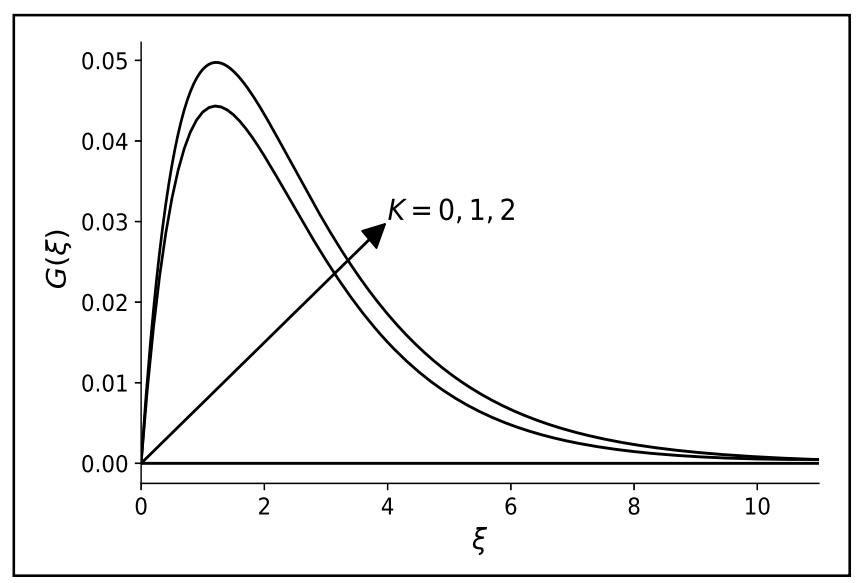

Figure 5. Microrotation profiles $G(\xi)$ of lower fluid for various $K$ when $\operatorname{Pr}=$ 7 and $\lambda=0.5$ under the effect of CHF.

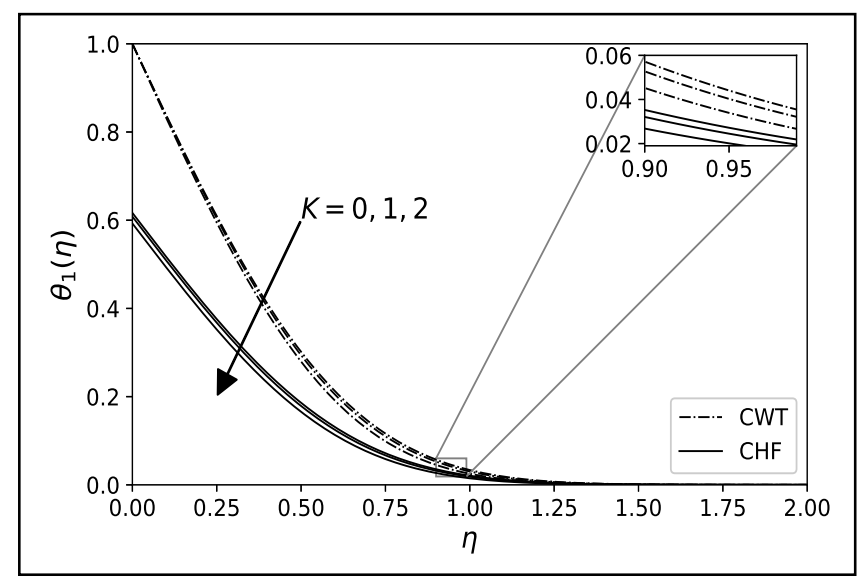

Figure 6. Temperature profiles $\theta_{1}(\eta)$ of upper fluid for various $K$ when $\operatorname{Pr}=$ 7 and $\lambda=0.5$.

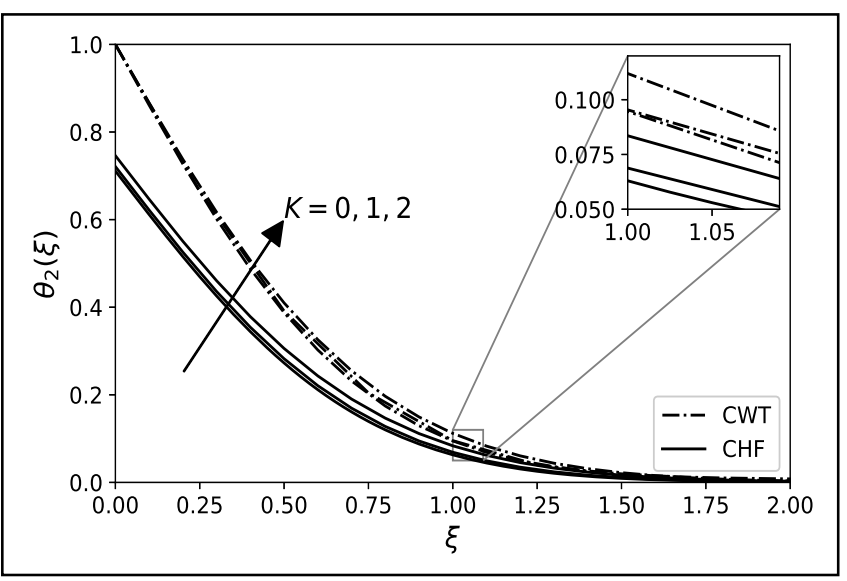

Figure 7. Temperature profiles $\theta_{2}(\xi)$ of lower fluid for various $K$ when $\operatorname{Pr}=$ 7 and $\lambda=0.5$.

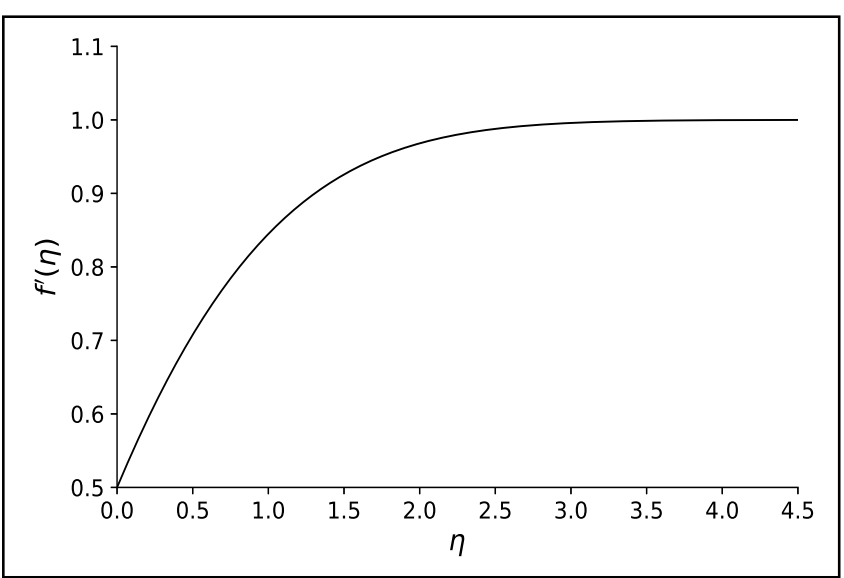

Figure 8. Velocity profiles $f^{\prime}(\eta)$ of upper fluid for various $\operatorname{Pr}$ when $K=1$ and $\lambda=0.5$ under the effect of CHF. 


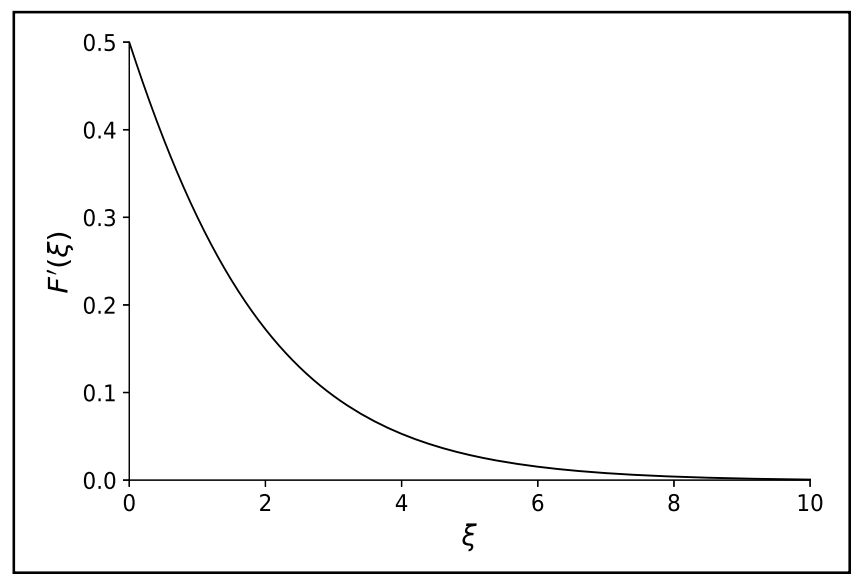

Figure 9. Velocity profiles $F^{\prime}(\xi)$ of lower fluid for various $\operatorname{Pr}$ when $K=1$ and $\lambda=0.5$ under the effect of CHF.

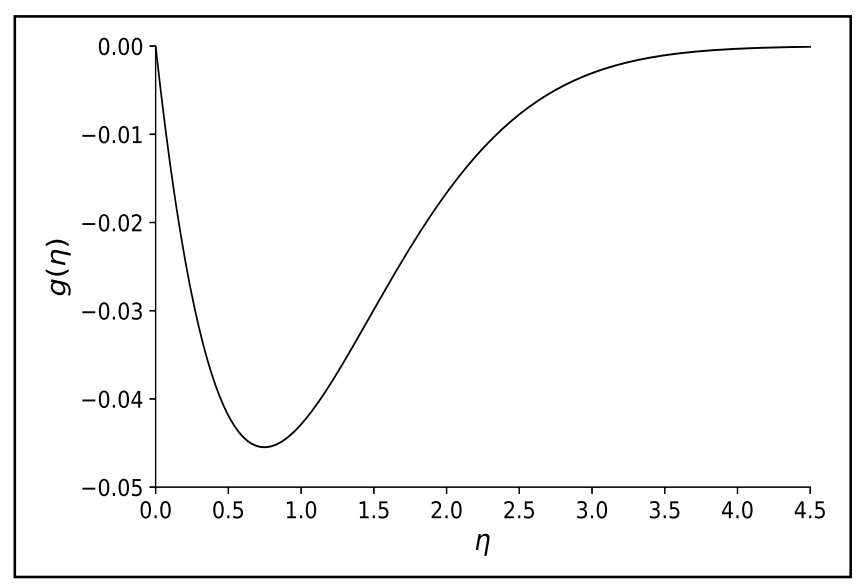

Figure 10. Microrotation profiles $g(\eta)$ of upper fluid for various $\operatorname{Pr}$ when $K=1$ and $\lambda=0.5$ under the effect of CHF.

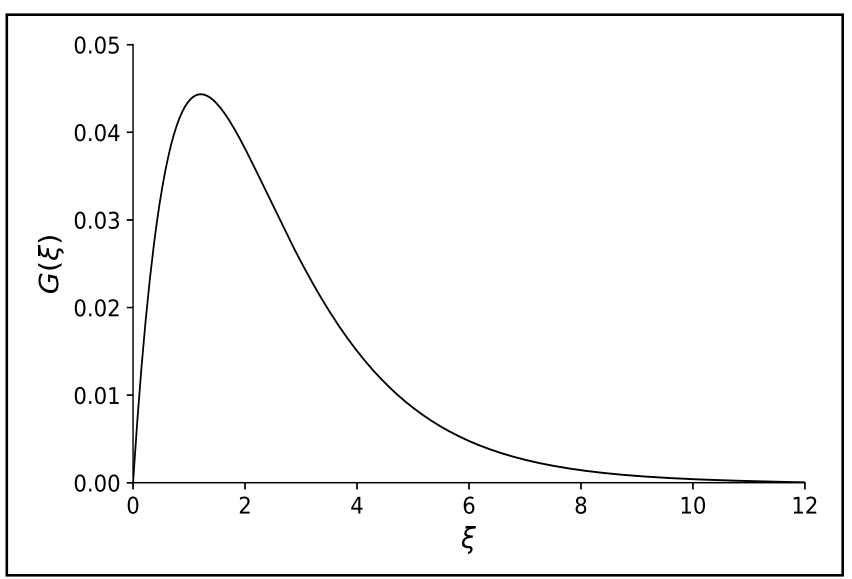

Figure 11. Microrotation profiles $G(\xi)$ of lower fluid for various $\operatorname{Pr}$ when $K=1$ and $\lambda=0.5$ under the effect of CHF.

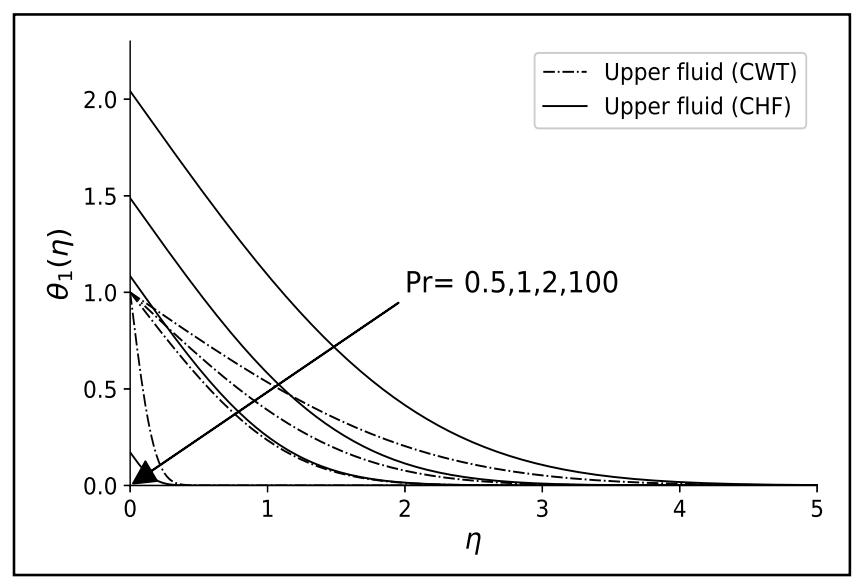

Figure 12. Temperature profiles $\theta_{1}(\eta)$ of upper fluid for various Pr when $K=1$ and $\lambda=0.5$.

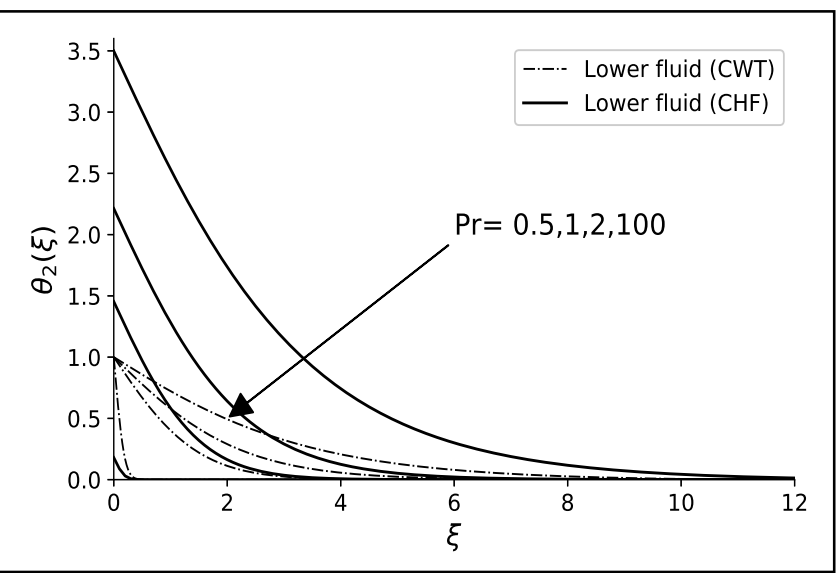

Figure 13. Temperature profiles $\theta_{2}(\xi)$ of lower fluid for various $\operatorname{Pr}$ when $K=1$ and $\lambda=0.5$. 


\section{Conclusion}

This study analyzed the effect of CHF on micropolar flow on a surface of another quiescent fluid. This problem was solved numerically using shooting method with Runge-Kutta-Gill in Python 3 software. The main points are summerized as follows:

- It is shown that, the temperature is higher in CHF compared to CWT at strecthing or shrinking parameter $\lambda=$ 0.5 .

- Meanwhile, as Pr increases, the temperature is decreasing for both CHF and CWT.

- Upper fluid and lower fluid has opposite trend with variation of micropolar parameter $K$.

\section{REFERENCES}

[1] A. C Eringen. Theory of micropolar fluids, Journal of Mathematics and Mechanics, Jul 1:1-8, 1966.

[2] T. Ariman, M. A. Turk \& N. D. Sylvester. Microcontinuum fluid mechanics- a review, International Journal Engineering Science, Vol. 11, 905-930, 1973.

[3] T. Ariman, M. A. Turk \& N. D. Sylvester. Applications of microcontinuum fluid mechanics, International Journal Engineering of Science, Vol. 12, No. 6, 273-293, 1974.

[4] A. J. Willson. Boundary layers in micropolar liquids, Mathematical Proceedings of the Cambridge Philosophical Society, 1970.

[5] G. Lukaszewicz. Micropolar fluids theory and applications, (N. Bellomo \& P. Torino, Eds.). Birkhäuser, 1999.

[6] V. A. Eremeyev, L. P. Lebedev, \& H. Altenbach. Foundations of micropolar mechanics, Springer Berlin Heidelberg, 2013

[7] D. A. S. Rees \& A. P. Bassom. The Blasius boundary layer flow of a micropolar fluid. International Journal of Engineering. Vol. 34, No. 1, 113-124. 1996.

[8] G. S. Guram, \& A. C. Smith. Stagnation flows of micropolar fluids with strong and weak interactions, Computers and Mathematics with Applications, Vol.6, No.2, 213-233, 1983.

[9] J. Peddieson. An application of the micropolar fluid model to the calculation of a turbulent shear flow, International Journal Engineering Science, Vol. 10, No. 1, 23-32, 1972.

[10] H. S. Takhar, \& V. Soundalgekar. Boundary layer flow of a micropolarfluid - non similar solution, Mechanics Research Communications, Vol. 13, No. 5, 255-263. 1986.

[11] A. Ishak, R. Nazar \& I. Pop. Boundary-layer flow of a micropolar fluid on a continuously moving or fixed permeable surface, International Journal of Heat and Mass Transfer, Vol. 50, No. 23-24, 4743-4748, 2007.
[12] S. Das \& S. K. Guha. Non-linear stability analysis of micropolar fluid lubricated journal bearings with turbulent effect, Industrial Lubrication and Tribology, 2018.

[13] H. A. Attia. Stagnation point flow and heat transfer of a micropolar fluid with uniform suction or blowing, Journal of the Brazilian Society of Mechanical Sciences and Engineering, Vol. 30 No. 1,57-65, (2008).

[14] A. C. Eringen. Theory of thermomicrofluids. Journal of Mathematical Analysis and Applications, Vol. 38, No. 2, 480-496, 1972.

[15] R. R. S. R. Gorla, R. Pender \& J. Eppichs. Heat transfer in micropolar boundary layer flow over a flat plate, International Journal Engineering of Science, Vol. 21, No. 7: 791-798. 1983.

[16] A. Ishak, R. Nazar and I.Pop. Flow and heat transfer characteristics on a moving flat plate in a parallel stream with constant surface heat flux, Heat and Mass Transfer, Vol. 45, No. 5, 563$567,2009$.

[17] C. Y. Wang. Stagnation flow on the surface of a quiescent fluid an exact solution of the Navier-Stokes Equation, Acta Technica, Vol.33, No. 2, 715-730. 1988.

[18] T. Liu . Nonorthogonal stagnation flow on the surface of a quiescent fluid - an exact solution of the Navier-Stokes, Quarterly of Applied Mathematics, Vol. 33, No. 2, 715-730, 1988.

[19] M. Reza, S. Panigrahi, \& A. K. Mishra. Stagnation point flow and heat transfer for a viscoelastic fluid impinging on a quiescent fluid, Academy Proceedings in Engineering Sciences, Vol. 42, No. 11, 1979-1986, 2017.

[20] M. Reza, \& A. S. Gupta. MHD stagnation-point flow of an electrically conducting fluid on the surface of another quiescent fluid, Acta Mechanica, Vol. 223, No. 11, 2303-2310. 2012.

[21] A. M. Rohni, Z. Omar, \& N. H.Man. Dual solutions of stagnation-point flow of a fluid on a shrinking surface of another quiescent fluid, In 4th Int'l Conference on Advances in Engineering Sciences \& Applied Mathematics (ICAESAM'2015), Vol. 2, 74-76, 2015.

[22] S. M. Isa, \& N. F. Mohammad, Boundary layer flow of dusty on a stretching sheet of another quiescent fluid, Journal of Physics: Conference Series, Vol. 819, No. 1, 2017.

[23] S. K. Jena \& M. N. Mathur. Similarity solutions for laminar free convection flow of a thermomicropolar fluid past a nonisothermal vertical flat plate, International Journal of Engineering Science, Vol. 19, No. 11, 1431 - 1439, 1981.

[24] G. Ahmadi. Self-similar solution of imcompressible micropolar boundary layer flow over a semi-infinite plate, International Journal of Engineering Science, Vol. 14, No. 7 ,639-646, 1976.

[25] G. Nath. Similar solutions for incompressible laminar boundary layer with pressure gradient in micropolar fluid, Rheologica Acta, Vol. 14, No.9, 850 - 875, 1975.

[26] R. Nazar, N. Amin, D. Filip, \& I. Pop, Stagnation point flow of a micropolar fluid towards a stretching sheet, International Journal of Non-Linear Mechanics, Vol. 39, No.7, 1227-1235, 2004. 
[27] D.A.S Rees. \& I. Pop. Free convection boundary layer flow of a micropolar fluid from a vertical flat plate, IMA Journal of Applied Mathematics (Institute of Mathematics \& its Applications), Vol. 61, No. 21, 179 - 197, 1998.

[28] Y.Y. Lok, N. Amin, \& I.Pop. Mixed convection near a nonorthogonal stagnation point flow on a vertical plate with uniform surface heat flux, Acta Mechanica, Vol. 186,No. 1-4,99, 2006.
[29] M.Z. Salleh,R. Nazar, \& I. Pop. Forced convection boundary layer flow at a forward stagnation point with Newtonian heating, Chemical Engineering Communication, Vol.196, No.9,987-996. 2009.

[30] E. R. G. Eckert. Die Berechnung des Wärmeüberganges in der laminaren Grenzschicht umströmter Körper, VDIForsch., 461, 1942. 